1 Universidade Federal da Paraíba (UFPB) - João Pessoa (PB), Brasil. elocruz17@hotmail.com

2 Universidade Federal da Paraíba (UFPB) - João Pessoa (PB), Brasil. isismilane@hotmail.com

3 Universidade Federal da Paraíba (UFPB), Departamento de Estatística, Programa de Pós-Graduação em Modelos de Decisão e Saúde - João Pessoa (PB) Brasil.

joaoagh@gmail.com

4 Universidade Federal da Paraíba (UFPB), Departamento de Estatística, Programa de Pós-Graduação em Modelos de Decisão e Saúde - João Pessoa (PB) Brasil.

hemilio@gmail.com

5 Universidade Federal da Paraíba (UFPB), Programa de Pós-graduação em Enfermagem e Programa de Pós-graduação em Modelos de Decisão em Saúde - João Pessoa (PB), Brasil.

srsantos207@gmail.com

\section{Implantação do e-SUS AB no Distrito Sanitário IV de João Pessoa (PB): relato de experiência}

\author{
Implementation of e-SUS AB in Sanitary District IV of João Pessoa \\ $(P B)$ : experience report
}

Ana Eloísa Cruz de Oliveira', Isis Milane Batista de Lima², João Agnaldo do Nascimento ${ }^{\mathbf{2}}$, Hemílio Fernandes Campos Coelho ${ }^{4}$, Sérgio Ribeiro dos Santos $\mathbf{5}$

RESUMO Os sistemas de informação têm o propósito de auxiliar os gestores nas tomadas de decisões. O e-SUS AB facilita e contribui para a organização do trabalho dos profissionais de saúde na Atenção Básica. O presente artigo tem o objetivo de apresentar o processo de implantação e desenvolvimento do e-SUS AB no Distrito Sanitário IV de João Pessoa (PB), em 2014. Trata-se de um estudo descritivo, na forma de relato de experiência, o qual aponta as dificuldades e qualidades do sistema. Conclui-se que os esforços para reestruturação do sistema só serão efetivos com o apoio dos envolvidos na implantação, na utilização e no aprimoramento do Sistema de Informação em Saúde da Atenção Básica (Sisab) e da estratégia e-SUS AB.

PALAVRAS-CHAVE Sistemas de informação; Atenção Primária à Saúde; Gestor de saúde.

ABSTRACT Information systems are intended to assist managers in decision making. The e-SUS $A B$ facilitates and contributes to the organization of the work of health professionals in Primary Care. This article aims to present the process of implementation and development of the e-SUS $A B$ in the Health District IV of João Pessoa (PB), in 2014. This is a descriptive study, in the form of experience report, which points out the difficulties and qualities of the system. We conclude that efforts to the restructuring of the system will only be effective with the support of those involved with the implementation, the use and improvement of the Information System of Primary Health Care (Sisab) and the strategy e-SUS AB.

KEYWORDS Information systems; Primary Health Care; Health manager. 


\section{Introdução}

A informação possui um papel estratégico dentro dos processos de tomada de decisão, no entanto, ainda é possível observar a existência de diversas dificuldades em acessar e tratar os dados de forma adequada, disponibilizando informações necessárias no processo de trabalho em saúde, em momento oportuno, bem como a falta de articulação que há entre os processos de planejamento e gestão da saúde com os próprios sistemas de informação (VASCONCELLOS ET AL., 2002).

Os sistemas de informação em saúde objetivam processar, armazenar, coletar e disseminar dados, auxiliando a gestão e possibilitando o aprimoramento das ações por ela desenvolvidas, uma vez que são capazes de oferecer suporte ao processo decisório em saúde (MARIN, 2010; CAVALCANTE; SILVA; FERREIRA, 2011). Para auxiliar os profissionais no planejamento, bem como na tomada de decisões relacionadas à gerência e à assistência aos pacientes, os sistemas devem disponibilizar informações adequadas, potencializar a comunicação e promover a segurança necessária no ambiente organizacional (CAVALCANTE ET AL., 2012).

Além disso, quando o sistema é utilizado de forma adequada, também pode promover a redução da dependência de papéis, principalmente, quando pode contar com um aparato tecnológico capaz de converter para a forma eletrônica as informações necessárias para o processo de trabalho, promovendo melhorias na atuação dos profissionais da saúde e, ainda, gerando redução de custos para a gestão (GUTIERREZ, 2011).

Logo, a informação é capaz de promover a aceleração do processo de identificação de problemas e de potencializar a resolubilidade das situações que venham a se apresentar, nos mais diversificados cenários. Inclusive, a informática se tornou o meio mais eficaz de disseminação dessa informação. Assim, reconhece-se a importância da informação e da informática no apoio à gestão do trabalho em saúde de forma cada vez mais significativa (BRASIL, 2012).

Desse modo, por meio da implantação de um sistema de informação em saúde, possibilita-se a comunicação entre profissionais e gestores, permitindo discussões, relacionamentos múltiplos, além de minimizar o impacto das barreiras culturais, da infraestrutura física e da distância, maximizando a troca de informações e a aquisição de conhecimentos que possam elevar a qualidade do cuidado prestado à população (SILVA, 2012).

Além disso, junto com a sociedade, os sistemas de informação em saúde também evoluem rapidamente. Com o surgimento das inovações tecnológicas, evoluem os conceitos, as formas de armazenamento, processamento e disseminação da informação, para que possam ser utilizadas da melhor forma possível pelos diversos públicos, diminuindo suas formas fragmentadas, algo ainda muito presente na realidade do Brasil (BRASIL, 2009).

Nessa perspectiva, o compromisso de reestruturar o Sistema de Informação da Atenção Básica (Siab) foi assumido pelo Departamento de Atenção Básica (DAB) da Secretaria de Atenção à Saúde (SAS), do Ministério da Saúde (MS), com o propósito de melhorar a qualidade da informação em saúde, otimizando o uso dessas informações pelos gestores, profissionais de saúde e cidadãos. Essa reestruturação é orientada por meio de diretrizes alinhadas com a Política Nacional de Atenção Básica (PNAB), a Política Nacional de Saúde Bucal, o Programa Nacional de Melhoria do Acesso e da Qualidade (PMAQ), o Programa Saúde na Escola (PSE), a Política Nacional de Práticas Integrativas e Complementares, a Política Nacional de Informação e Informática em Saúde (PNIIS), o Plano Estratégico de e-Saúde no Brasil e a integração aos sistemas de informação que compõem as Redes de Atenção à Saúde (RAS) (BRASIL, 2014B).

Assim, com o intuito de desenvolver, reestruturar e garantir a integração dos sistemas de informação, de modo a permitir um 
registro da situação de saúde individualizado por meio do Cartão Nacional de Saúde, surge a estratégia e-SUS AB, do MS (BRASIL, 2013). O ponto inicial dessa estratégia é o registro das informações em saúde de forma individualizada, para que seja possível realizar um futuro acompanhamento do histórico de atendimentos de cada usuário, assim como da produção de cada profissional da Atenção Básica (AB). O e-SUS AB ainda traz consigo a integração dos diversos sistemas de informação oficiais existentes na $\mathrm{AB}$, reduzindo a necessidade de registrar informações similares em mais de um instrumento (fichas/sistemas), o que otimiza o trabalho dos profissionais, o uso da informação para a gestão e qualificação do cuidado em saúde (BRASIL, 2014A).

O período de transição do Siab para alimentar o novo Sistema de Informação em Saúde da Atenção Básica (Sisab) iniciou-se a contar da data de publicação da Portaria GM/MS no ${ }^{0} 412$, de 10 de Julho de 2013, com duração de um ano. No entanto, em virtude do andamento das implantações do e-SUS $\mathrm{AB}$ nos municípios brasileiros, surgiu a necessidade de adequação desse prazo de transição, na $5^{\mathrm{a}}$ Reunião Ordinária da Comissão Intergestores Tripartite (CIT), realizada no dia 31 de Julho de 2014, e, com isso, tornou-se obrigatório o envio de informações para a base de dados do Sisab, a partir da competência de junho de 2015 , sendo tais dados exportados até o final do mês de julho de 2015 (BRASIL, 2014B).

A implantação do e-SUS AB representa um importante avanço na qualificação e no uso da informação registrada durante as ações de saúde desenvolvidas na AB. Como em todo momento de mudança, há um período inicial mais crítico até que os novos fluxos e instrumentos utilizados sejam incorporados na rotina dos profissionais das equipes de saúde. Desde a instituição do Sisab, em julho de 2013, muitos municípios já avançaram nessa implantação, porém, outros ainda necessitam de suporte e/ou de um acompanhamento mais próximo, considerando que o Siab deixará de existir e que há a necessidade de acelerar a implantação do e-SUS AB, de modo que as equipes possam adequar seus processos de trabalho antes do prazo final da transição, garantindo a consistência e a qualidade das informações que subsidiarão a produção dos indicadores de saúde e das demais ferramentas de gestão da informação (BRASIL, 2014A).

Portanto, o presente artigo tem o objetivo de apresentar a experiência das autoras no processo de implantação e desenvolvimento do e-SUS AB no Distrito Sanitário IV (DS IV) do município de João Pessoa, na Paraíba, pontuando aspectos do processo e esperando subsidiar a reflexão sobre a implantação de um novo modelo de sistema de informação, promovendo um melhor desenvolvimento do mesmo e otimizando sua utilização nos municípios que estão implantando ou ainda implantarão o e-SUS AB.

\section{Relato da experiência}

O nome e-SUS AB faz referência a um SUS eletrônico, cujo objetivo é facilitar e contribuir para a organização do trabalho dos profissionais de saúde, elemento decisivo para a qualidade da atenção à saúde prestada à população. Os frutos dessa nova estratégia são o Sisab e um novo software, o e-SUS $\mathrm{AB}$, que possui duas versões: o Prontuário Eletrônico do Cidadão (PEC) e a Coleta de Dados Simplificada (CDS) (BRASIL, 2013).

A diferença básica entre as versões existentes é que o PEC permite que o profissional possa inserir as informações de atendimento no momento em que está realizando a consulta, o procedimento ou até mesmo uma visita domiciliar. Já o CDS utiliza oito impressos: ficha de atendimento individual, ficha de atendimento odontológico, ficha de procedimentos, ficha de cadastro individual, ficha de cadastro domiciliar, ficha de visita domiciliar, ficha de atividade coletiva e ficha 
de marcadores de consumo alimentar. Ao realizar o atendimento, cada profissional preenche a ficha destinada para o tipo de ação desenvolvida, envia ao DS para que possa ser digitada, alimentando o sistema, para, assim, retornar ao serviço, onde poderá ser arquivada.

A partir do mês de outubro de 2013, foram realizadas reuniões das referências da gestão da informação nos cinco DS, juntamente com a Gerência de Atenção Básica da Secretaria Municipal de Saúde, com o intuito de implantar o e-SUS AB em João Pessoa (PB).

Após ampla discussão sobre o novo Sistema de Informação da Atenção Básica, teve início o processo de implantação, com o cadastro do gestor no sistema de controle de uso do e-SUS AB e a identificação dos recursos disponíveis, para que se pudesse escolher o sistema a ser implantado. Com base nesse levantamento da capacidade tecnológica disponível na Secretaria Municipal de Saúde e nas Unidades de Saúde da Família (computadores, impressoras, internet, ambientes etc.), a indicação do sistema a ser adotado foi a Coleta de Dados Simplificada (CDS). Embora o e-SUS $A B$ tenha a versão $P E C$, a versão CDS foi escolhida em João Pessoa (PB), pelo menos até que o município possa se estruturar de forma adequada para a instalação do PEC em todas as Unidades de Saúde, o que requer mais tempo e investimentos.

Com as informações do sistema de controle de uso do e-SUS AB, o CDS foi instalado nos cinco DS e na Secretaria de Saúde, já que ele precisa, obrigatoriamente, de uma instalação centralizadora municipal ou PEC para transmitir dados para a base federal. Mesmo com o uso do CDS, a estratégia avança ao permitir a entrada dos dados orientada pelo curso natural do atendimento, e não focada na situação-problema de saúde. A entrada de dados individualizada por cidadão abre caminho para a gestão do cuidado e para a aproximação desses dados ao processo de planejamento da equipe.

Assim, seguindo o processo de implantação, foi elaborado um cronograma de capacitação e sensibilização dos profissionais da AB. Cada distrito planejou e executou um ciclo de capacitações para a utilização do e-SUS AB e capacitação dos profissionais: médicos, enfermeiros, técnicos de enfermagem, odontólogos, auxiliares em saúde bucal e Agentes Comunitários de Saúde (ACS), que foram divididos em turmas, de acordo com a categoria profissional e o quantitativo de pessoas. Foram momentos de exposição de todas as fichas que seriam utilizadas pelos profissionais, sendo cada campo explicado de forma detalhada. Nessa ocasião, os participantes tiveram a oportunidade de esclarecer dúvidas existentes e também foram distribuídos manuais do e-SUS AB para cada equipe de saúde. Os profissionais do Núcleo de Apoio à Saúde da Família (Nasf) também tiveram um momento de capacitação, já que os mesmos utilizarão o e-SUS AB em suas atividades.

Após a finalização das capacitações realizadas durante o mês de abril de 2014, foi realizada a distribuição das fichas em cada unidade de saúde, para que, em maio de 2014, as Unidades de Saúde do DS IV pudessem trabalhar com o novo sistema.

Assim, com o uso das fichas, foi necessário realizar o cadastro dos usuários de cada território. Essa estratégia de cadastramento foi desenhada para ser implantada de maneira gradual. Ao mesmo tempo que os ACS realizavam o cadastro, os demais profissionais da equipe desenvolviam suas ações. Lembrando que, durante todo esse processo, foi essencial sensibilizar os usuários quanto à importância do Cartão Nacional de Saúde (CNS), uma vez que a identificação do registro nos atendimentos por meio do CNS permite uma efetiva ordenação e uma gestão do cuidado do cidadão, além da possibilidade de compartilhamento de informações com outros serviços de saúde.

Alguns sistemas de informação utilizados pela $\mathrm{AB}$ foram desativados com a chegada do e-SUS AB, que, por sua vez, veio integrá-los, 
como foi o caso do Siab, do Hiperdia e do Sistema de Vigilância Alimentar Nutricional (Sisvan), com projetos de integrar também o Sistema de Acompanhamento do Programa de Humanização no Pré-Natal e Nascimento (Sisprenatal) em futuras atualizações. Foram retirados das unidades impressos que não seriam mais utilizados pelas equipes, e os digitadores responsáveis pelos sistemas desativados formaram a equipe de digitadores responsáveis pela alimentação do e-SUS AB.

Assim, com o início da implantação do e-SUS, as dúvidas foram surgindo com relação à utilização das fichas e puderam ser esclarecidas por meio de um guia ou manual distribuído na capacitação, de telefonemas dos profissionais das equipes e dos apoiadores ao DS IV e da presença da referência da gestão da informação em visitas técnicas às unidades, assim como em algumas reuniões de equipe. À medida que as fichas chegavam ao DS IV para digitação, também era possível detectar variados erros de preenchimento, alguns que até impediam a digitação por se tratar de campos obrigatórios no sistema.

Em decorrência desse fato, foram elaborados dois impressos, e com esses instrumentos tornou-se possível sinalizar para as equipes as fichas do e-SUS AB que foram digitadas com êxito, e que já poderiam ser arquivadas no serviço, e aquelas que possuíam alguma pendência a ser resolvida, para que a mesma pudesse ser solucionada e a ficha pudesse retornar ao DS IV para ser inserida no sistema com sucesso. Ambos foram apresentados aos demais DS em reunião na Secretaria Municipal de Saúde, padronizando o instrumento para todos os outros distritos.

O início da alimentação do sistema ocorreu de forma lenta pelos seguintes motivos:

Iniciar a utilização das fichas com um quantitativo de impressos insuficiente frente à necessidade de cada equipe de saúde;

Ser um período de adaptação para os digitadores;
Encontrar erros nas fichas enviadas pelos profissionais das Unidades de Saúde;

Alguns computadores apresentarem problemas técnicos, necessitando de reparos.

Todas as dificuldades enfrentadas durante a implantação foram relatadas no relatório solicitado pela Diretoria de Atenção Básica, para que o desempenho do sistema pudesse ser otimizado.

Logo, a primeira exportação de dados no DS IV foi realizada no dia 29 de maio de 2014, sendo constatada deficiência na oferta de dados presentes no único relatório gerado pelo sistema, que era composto apenas pela quantidade de fichas digitadas por máquina, divididas apenas pelos tipos de fichas.

Nos meses seguintes, foi observado que o DS IV já estava com um quantitativo suficiente de fichas fornecidas pela Secretaria de Saúde, seus digitadores já estavam adaptados ao funcionamento do e-SUS AB, os computadores foram reparados, sendo alguns substituídos por novos, e os erros cometidos pelos profissionais no preenchimento das fichas foram diminuindo de forma significativa.

Algo que passou a constituir uma situação problema foi a demanda reprimida de fichas para digitação. Com a implantação e com todos os ACS realizando o cadastramento das suas áreas, o número de fichas que chegava ao setor de digitação acabou sendo maior que o número de fichas que eram digitadas e encaminhadas de volta aos serviços, principalmente quando em concomitância com as fichas de atendimentos, procedimentos, visitas domiciliares e atividades coletivas.

Por ser uma dificuldade não só do DS IV, mas de todos os distritos de João Pessoa, foi realizado um plano operativo para dar vazão às fichas de cadastramento, no qual os DS puderam formar grupos de pessoas para auxiliar em uma digitação extra, feita com o apoio da Universidade Federal da Paraíba, que cedeu o laboratório de informática do Centro de Ciências da Saúde para que tal 
atividade fosse desenvolvida, resultando em uma importante contribuição no processo de diminuição da demanda reprimida de fichas a digitar.

Atualmente, as maiores limitações que o sistema apresenta são a impossibilidade de edição de dados já digitados e exportados no e-SUS AB e a escassez de dados fornecidos no relatório de exportação, que ainda não passou por nenhuma modificação significativa que fizesse com que o mesmo ofertasse uma maior quantidade de dados da situação de saúde dos territórios, não oferecendo ferramentas necessárias para o processo de monitoramento e processamento de dados pelos gestores e, consequentemente, dificultando o processo de planejamento e avaliação de ações realizadas na $\mathrm{AB}$, tanto no âmbito do DS IV como no da Secretaria Municipal de Saúde.

Espera-se que, em breve, nas próximas atualizações do e-SUS AB, as limitações possam ser resolvidas ou amenizadas, melhorando seu processo de utilização, favorecendo o processo de gestão da informação na AB de João Pessoa e elevando ainda mais a qualidade dos serviços de saúde ofertados pelo SUS.

\section{Considerações finais}

Com o passar dos anos, com as transformações que a sociedade sofre e as mudanças apresentadas pela situação de saúde da população, faz-se necessário que os sistemas de informação em saúde acompanhem toda essa dinâmica realidade, sendo modificados, aprimorados e modernizados com base nas inovações tecnológicas cada vez mais avançadas e presentes na gestão da informação.

O processo de implantação de um sistema de informação é complexo, principalmente quando é necessário realizar a transição de sistemas já utilizados há muitos anos para um novo sistema que traz consigo uma nova proposta de utilização, com ferramentas diferenciadas.

É notório que todos os esforços de reestruturação do sistema só serão completos e efetivos com o envolvimento real dos gestores, dos profissionais de saúde e dos trabalhadores do SUS na implantação, na utilização e no aprimoramento do Sisab e da estratégia e-SUS AB, uma vez que esse é um processo contínuo, que exige tempo e muitos estudos para que possa ser, na prática, tudo aquilo que foi idealizado na teoria, em seus manuais, adaptando-se da melhor forma a cada realidade, principalmente quando se trata de um território diversificado como o Brasil, com tantas particularidades.

Em João Pessoa (PB), a reestruturação dos sistemas de saúde na AB junto ao e-SUS $A B$ está avançada e, embora seja algo novo, já mostrou benefícios no processo de trabalho dos profissionais envolvidos, principalmente na diminuição da quantidade de impressos utilizados nos registros de atendimentos e procedimentos realizados por cada um. Apesar de ainda apresentar limitações, como é o caso da impossibilidade de edição das informações já alimentadas no sistema e o fato de possuir um relatório que pouco oferece subsídios para monitoramento da situação de saúde dos territórios, espera-se que seja algo resolvido com o surgimento das futuras versões do sistema.

Tal relato de experiência foi realizado com o intuito não simplesmente de expor o processo de implantação do e-SUS AB em João Pessoa (PB), mas, também, de servir de base no processo de implantação do sistema em outros municípios e de conhecimento de gestores, profissionais e da população em geral, uma vez que a temática ainda se faz pouco presente na literatura, já que diz respeito a um sistema de informação recentemente criado e utilizado. 


\section{Referências}

BRASIL. Ministério da Saúde. A experiência brasileira em sistemas de informação em saúde. Brasília, DF: Editora do Ministério da Saúde, 2009.

Ministério da Saúde. Política de Informação e Informática em Saúde. Brasília, DF, 2004. Disponível em: <http://bvsms.saude.gov.br/bvs/publicacoes/ PoliticaInformacaoSaude29_03_2004.pdf $>$. Acesso em: 15 out. 2014.

Ministério da Saúde. Secretaria de Atenção à Saúde. e-SUS AB Atenção Básica: Sistema com Coleta de Dados Simplificada: CDS. Brasília, DF: Editora do Ministério da Saúde, 2013. Disponível em: <http://189.28.128.100/dab/docs/portaldab/ documentos/manual_cds.pdf $>$. Acesso em: 9 out. 2014

Ministério da Saúde. Secretaria de Atenção à Saúde. e-SUS AB Atenção Básica: Manual do Sistema com Coleta de Dados Simplificada: CDS. Brasília, DF, 2014a. Disponível em: <http://189.28.128.100/dab/docs/ portaldab/documentos/manual_CDS_ESUS_1_3_0. pdf $>$. Acesso em: 10 jan. 2015

Ministério da Saúde. Secretaria de Atenção à Saúde. Nota técnica DAB/SAS/MS: Esclarecimentos e orientações sobre a estratégia e-SUS AB. Brasília, DF, 2014b. Disponível em: <http://189.28.128.100/dab/docs/ portaldab/documentos/nota_tecnica_prorrogacao_ eSUS-AB.pdf $>$. Acesso em: 11 jan. 2015.

CAVALCANTE, R. B.; SILVA, P. C.; FERREIRA, M. N. Sistema de informação em saúde: possibilidades e desafios. Revista Enfermagem da UFSM, Santa Maria, v. 1, n. 2, p. 290-9, 2011.
CAVALCANTE, R. B. et al. Sistema de Informação Hospitalar: utilização no processo decisório. J. Health Inform., São Paulo, v. 4, n. 3, p. 73-9, 2012.

GUTIERREZ, M. A. Sistemas de informação hospitalares: progressos e avanços. J. Health Inform., São Paulo, v. 3, n. 2, p. 17-23, 2011.

MARIN, H. F. Sistemas de informação em saúde: considerações gerais. J. Health Inform., São Paulo, v. 2, n. 1, p. 24-8, 2010.

SILVA, L. M. Sistema de Informação: instrumento para qualificação da gestão do relatório de auditoria médica. 2012. 31 f. Trabalho de Conclusão de Curso (Especialização em Informação Científica e Tecnológica em Saúde) - Fundação Oswaldo Cruz, Porto Alegre, 2012.

VASCONCELLOS, M. M.; MORAES, I. H.; CAVALCANTE, M. T. L. Política de saúde e potencialidades de uso das tecnologias da informação. Saúde em Debate, Rio de Janeiro, v. 26, n. 61, p. 219-235, 2002.

Recebido para publicaç̃o em outubro de 2015

Versão final em maio de 2016

Conflito de interesses: inexistente

Suporte financeiro: não houve 